\title{
Chiral two- and three-nucleon forces along medium-mass isotope chains
}

\author{
V. Somà,${ }^{1,2,3,{ }^{*}}$ A. Cipollone, ${ }^{4}$ C. Barbieri, ${ }^{4, \dagger}$ P. Navrátil,${ }^{5}$ and T. Duguet ${ }^{3,6, \ddagger}$ \\ ${ }^{1}$ Institut für Kernphysik, Technische Universität Darmstadt, 64289 Darmstadt, Germany \\ ${ }^{2}$ ExtreMe Matter Institute EMMI, GSI Helmholtzzentrum für Schwerionenforschung GmbH, 64291 Darmstadt, Germany \\ ${ }^{3}$ CEA-Saclay, IRFU/Service de Physique Nucléaire, 91191 Gif-sur-Yvette, France \\ ${ }^{4}$ Department of Physics, University of Surrey, Guildford GU2 7XH, United Kingdom \\ ${ }^{5}$ TRIUMF, 4004 Westbrook Mall, Vancouver, British Columbia, Canada V6T 2A3 \\ ${ }^{6}$ National Superconducting Cyclotron Laboratory and Department of Physics and Astronomy, \\ Michigan State University, East Lansing, Michigan 48824, USA
}

(Received 8 December 2013; revised manuscript received 15 April 2014; published 5 June 2014)

\begin{abstract}
$A b$ initio calculations have shown that chiral two- and three-nucleon interactions correctly reproduce binding energy systematics and neutron drip lines of oxygen and nearby isotopes. Exploiting the novel Gorkov-Green's function approach applicable to genuinely open-shell nuclei, we present the first ab initio investigation of Ar, K, $\mathrm{Ca}, \mathrm{Sc}$, and $\mathrm{Ti}$ isotopic chains. In doing so, stringent tests of internucleon interaction models are provided in the medium-mass region of the nuclear chart. Leading chiral three-nucleon interactions are shown to be mandatory to reproduce the trend of binding energies throughout these chains and to obtain a good description of two-neutron separation energies. At the same time, nuclei in this mass region are systematically overbound by about $40 \mathrm{MeV}$. While the fundamental $N=20$ and 28 magic numbers do emerge from basic internucleon interactions, the former is shown to be significantly overestimated, which points to deficiencies of state-of-the-art chiral potentials. The present results demonstrate that ab initio many-body calculations can now access entire medium-mass isotopic chains including degenerate open-shell nuclei and provide a critical testing ground for modern theories of nuclear interactions.
\end{abstract}

DOI: 10.1103/PhysRevC.89.061301

PACS number(s): 21.60.De, 21.30.-x, 21.45.Ff, 27.40.+z

Introduction. Many-body interactions involving more than two nucleons have been long known to play an important role in nuclear physics. They arise naturally, due to the internal structure of the nucleon, and are deemed to be necessary to explain saturation properties of nucleonic matter [1-3]. In finite systems, three-nucleon forces (3NFs) provide key mechanisms governing, e.g., the shell evolution and the boundaries of nuclear stability. Studies based on interactions derived from chiral effective field theory (EFT) [4] have shown that leading two-pion 3NF terms (of the Fujita-Miyazawa type) induce changes in the location of traditional magic numbers and explain the anomalous position of the oxygen neutron drip line compared to neighboring elements [5-7].

$A b$ initio many-body methods currently capable of targeting nuclei beyond the oxygen region include selfconsistent Green's function (SCGF) [7,8], coupled-cluster (CC) $[9,10]$, and in-medium similarity renormalization group (IM-SRG) [11,12] theories. These approaches make use of sophisticated and accurate many-body schemes that have, however, been intrinsically limited, until recently, to the (vicinity of) closed-shell systems. Thus, ab initio calculations have been confined so far to closed-(sub)shell nuclei along isotopic chains in the oxygen mass region $[6,7,13]$ and to heavier, isolated, closed-shell systems $[12,14,15]$. To overcome this limitation and address genuinely open-shell nuclei, a novel method based on the Gorkov reformulation of SCGF

\footnotetext{
*vittorio.soma@cea.fr

†C.Barbieri@surrey.ac.uk

${ }^{\ddagger}$ thomas.duguet@cea.fr
}

theory has been introduced [16] along with proof-of-principle calculations $[17,18]$. Based on this approach, the present work constitutes the first-ever $a b$ initio application to the description of medium-mass open-shell nuclei starting from realistic two- and three-nucleon interactions. Specifically, we address five isotopic chains around $Z=20$, namely, $\mathrm{Ar}, \mathrm{K}, \mathrm{Ca}$, $\mathrm{Sc}$, and Ti. This extends the systematic and model-independent description of nuclei beyond the light sector of the nuclear chart, opening up a new region where chiral interactions can be tested and fundamental questions such as the emergence of magic numbers from basic nuclear forces can be addressed.

Our results demonstrate that leading chiral $3 N$ interactions are key to reproducing the trend of binding energies but nuclei are systematically overbound in contrast to what has been seen around oxygen [6,7]. Despite this overbinding, relative energies (specifically two-neutron separation energies) are fairly well reproduced once $3 \mathrm{NFs}$ are accounted for. Additionally, $N=20$ and 28 magic numbers do emerge, the latter greatly benefiting from the inclusion of $3 \mathrm{NFs}[5,19]$. Still, the magic character of the $N=20$ neutron number is significantly overestimated, which points to deficiencies of state-of-the-art chiral nuclear interactions.

Formalism. We start from the intrinsic Hamiltonian $\hat{H}_{\text {int }}=$ $\hat{T}-\hat{T}_{\text {c.m. }}+\hat{V}+\hat{W}$, with the kinetic energy of the center of mass subtracted and $\hat{V}$ and $\hat{W}$ being the two-nucleon $(N N)$ and $3 N$ interactions. The Gorkov formalism exploits the breaking of particle-number symmetry to effectively account for the nonperturbative physics associated with pairing correlations. Specifically, it targets the ground state, $\left|\Psi_{0}\right\rangle$, of the grand canonical Hamiltonian $\hat{\Omega}_{\text {int }}=\hat{H}_{\text {int }}-\mu_{p} \hat{Z}-\mu_{n} \hat{N}$ under the constraint that the correct particle number $A=N+Z$ is 
recovered on average: $Z=\left\langle\Psi_{0}|\hat{Z}| \Psi_{0}\right\rangle$ and $N=\left\langle\Psi_{0}|\hat{N}| \Psi_{0}\right\rangle$. The many-body Schrödinger equation is transformed into the Gorkov equation,

$$
\left.\left(\begin{array}{cc}
T+\Sigma^{11}(\omega)-\mu_{k} & \Sigma^{12}(\omega) \\
\Sigma^{21}(\omega) & -T+\Sigma^{22}(\omega)+\mu_{k}
\end{array}\right)\right|_{\omega_{k}}\left(\begin{array}{l}
\mathcal{U}^{k} \\
\mathcal{V}^{k}
\end{array}\right)=\omega_{k}\left(\begin{array}{l}
\mathcal{U}^{k} \\
\mathcal{V}^{k}
\end{array}\right)
$$

whose solutions are the poles of the single-nucleon propagators, $\omega_{k} \equiv \Omega_{k}-\Omega_{0}$, where the index $k$ refers to normalized eigenstates of $\hat{\Omega}_{\text {int }}$ that fulfill $\hat{\Omega}_{\text {int }}\left|\Psi_{k}\right\rangle=\Omega_{k}\left|\Psi_{k}\right\rangle$, and the probability amplitudes $\mathcal{U}^{k}\left(\mathcal{V}^{k}\right)$ to reach state $\left|\Psi_{k}\right\rangle$ by adding (removing) a nucleon to (from) $\left|\Psi_{0}\right\rangle$. The self-energy splits into static (first-order) and dynamic terms, $\Sigma(\omega)=\Sigma^{(\infty)}+$ $\Sigma^{(\mathrm{dyn})}(\omega)$. In the present work we consider all first- and secondorder contributions, which define the many-body truncation of the method.

The inclusion of 3NFs in standard SCGF formalism is discussed in depth in Ref. [20], with first applications in Refs. [1,7,20]. Here we improve on the prescription of Ref. [7] to extend the Gorkov approach $[16,18]$ to $3 \mathrm{NFs}$ for the first time. The second-order self-energy contains only interaction-irreducible diagrams and it is calculated using an effective $N N$ interaction, which includes contributions from $\hat{W}$. However, the static self-energy acquires extra terms from interaction-reducible diagrams involving $3 \mathrm{NFs}$ that lead to the following corrections:

$$
\begin{aligned}
& \Delta \Sigma_{\alpha \beta}^{11,(\infty)}=-\left[\Delta \Sigma_{\bar{\alpha} \bar{\beta}}^{22,(\infty)}\right]^{*}=\frac{1}{2} \sum_{\gamma \delta \mu \nu} W_{\alpha \gamma \delta, \beta \mu \nu} \rho_{\mu \gamma} \rho_{\nu \delta}, \\
& \Delta \Sigma_{\alpha \beta}^{12,(\infty)}=\left[\Delta \Sigma_{\beta \alpha}^{21,(\infty)}\right]^{*}=\frac{1}{2} \sum_{\gamma \delta \mu \nu} W_{\alpha \bar{\beta} \delta, \mu \bar{\nu} \gamma} \tilde{\rho}_{\mu \nu} \rho_{\gamma \delta},
\end{aligned}
$$

where greek indices $\alpha, \beta, \ldots$ label a complete orthonormal single-particle basis (barred quantities refer to time-reversed states) and $\rho(\tilde{\rho})$ denotes the normal (anomalous) one-body density matrix [16].

Starting from a Hatree-Fock-Bogoliubov reference state we follow the sc0 prescription discussed in Ref. [18]; i.e. $\Sigma^{(\mathrm{dyn})}$ remains unchanged throughout the calculation while $\Sigma^{(\infty)}$ is computed in a self-consistent fashion. A new effective one-body potential is generated at each iteration using correlated density matrices, which allows one to go beyond the standard normal-order two-body approximation for threenucleon forces. Once convergence is reached, the total energy is calculated through the Koltun sum rule corrected for the presence of 3NFs [7,20],

$$
E_{0}^{A}=E_{0}^{A(N N)}-\frac{1}{2}\left\langle\Psi_{0}|\hat{W}| \Psi_{0}\right\rangle,
$$

where $E_{0}^{A(N N)}$ represents the energy sum rule for $N N$ interactions only, adapted to the Gorkov framework [16]. The expectation value of $\hat{W}$ is obtained at first order in terms of the correlated normal density matrix

$$
\left\langle\Psi_{0}|\hat{W}| \Psi_{0}\right\rangle \approx \frac{1}{6} \sum_{\alpha \beta \gamma \mu \nu \xi} W_{\alpha \beta \gamma, \mu \nu \xi} \rho_{\mu \alpha} \rho_{\nu \beta} \rho_{\xi \gamma} .
$$

The contribution containing two anomalous density matrices was checked to be negligible and hence is not included here.
The present formalism assumes a $J^{\Pi}=0^{+}$ground state and therefore targets even-even systems. The ground-state energy of odd-even neighbors is obtained through $[16,21]$

$$
E_{0}^{A}=\widetilde{E}^{A}+\omega_{k=0}, \quad \text { for } A \text { odd },
$$

where $\widetilde{E}^{A}$ is the energy of the odd-even nucleus computed as if it were an even-even one, i.e., as a fully paired evennumber-parity state but forced to have an odd number of particles on average, while $\omega_{k=0}$ denotes the lowest pole energy extracted from Eq. (1) for that calculation. Equation (5) is potentially exact such that its ability to account for blocking and polarization effects (and beyond) only depends on the scheme used to truncate the self-energy expansion.

Results. Calculations were performed using chiral $N N$ and $3 \mathrm{~N}$ forces evolved to low-momentum scales through freespace similarity renormalization group (SRG) techniques [22]. The original $N N$ interaction was generated at next-tonext-to-next-to-leading order $\left(\mathrm{N}^{3} \mathrm{LO}\right)$ with cutoff $\Lambda_{2 N}=$ $500 \mathrm{MeV}$ [23,24], while a local $\mathrm{N}^{2} \mathrm{LO} 3 \mathrm{NF}$ [25] with a reduced cutoff of $\Lambda_{3 N}=400 \mathrm{MeV}$ was employed. The $3 \mathrm{NF}$ low-energy constants $c_{D}=-0.2$ and $c_{E}=0.098$ were fitted to reproduce ${ }^{4} \mathrm{He}$ binding energy [10]. The SRG evolution on the sole chiral $N N$ interaction already generates $3 N$ operators in the Hamiltonian, which we refer hereafter to as the "induced" 3NF. When the pre-existing chiral $3 N$ interaction, including the two-pion exchange Fujita-Miyazawa contribution, is included, we refer to the "full" 3NF. Calculations were performed in model spaces up to 14 harmonic oscillator (HO) shells $\left[N_{\max } \equiv \max (2 n+l)=13\right]$, including all $N N$ matrix elements and limiting $3 \mathrm{NF}$ ones to configurations with $N_{1}+N_{2}+N_{3} \leqslant N_{\max }^{3 \mathrm{NF}}=16$. An SRG cutoff $\lambda=2.0 \mathrm{fm}^{-1}$ was used.

Figure 1 shows the ${ }^{51} \mathrm{~K}$ binding energy as a function of the model space size and the HO frequency used. Being one of the heaviest nuclei considered here, ${ }^{51} \mathrm{~K}$ is representative of the slowest convergence obtained in this work. Changing the model space from $N_{\max }=12$ to 13 lowers its groundstate energy by $2.1 \mathrm{MeV}$, which corresponds to about $0.5 \%$ of the total binding energy. This is much smaller than the uncertainties resulting from truncating the many-body expansion of the self-energy at second order (see below).

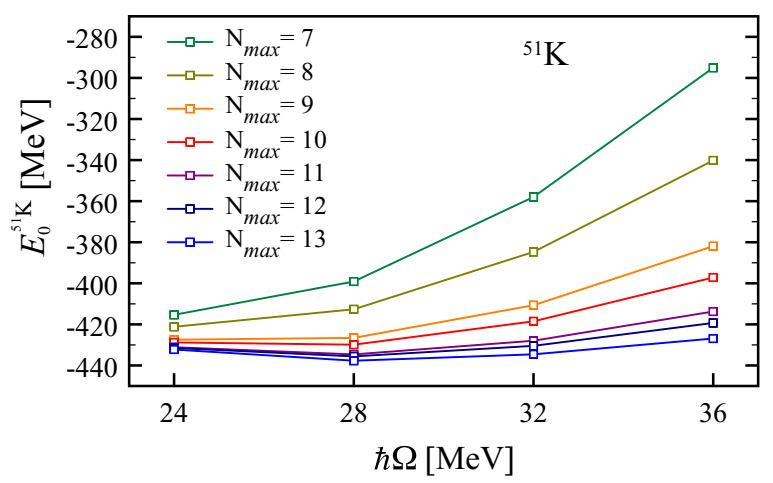

FIG. 1. (Color online) Convergence of the binding energy of ${ }^{51} \mathrm{~K}$ with respect to the basis size and HO frequency, for the full Hamiltonian. 
Other isotopes have similar speeds of convergence, e.g., the same variation of the model space induces a change of $1 \mathrm{MeV}$ in ${ }^{40} \mathrm{Ca}$. Thus, one expects convergence errors to cancel to a large extent when calculating two-neutron separation energies $S_{2 n} \equiv E_{0}^{Z, N}-E_{0}^{Z, N-2}$. To test this we performed exponential extrapolations of the calculated binding energies of a few nuclei, using the last few odd values of $N_{\max }$. We found variations of at most $\approx 500 \mathrm{keV}$ with respect to the value calculated at $N_{\max }=13$. Hence, we take this as an estimate of the convergence error on computed $S_{2 n}$. In the following we present our results calculated for $N_{\max }=13$ and $\hbar \Omega=$ $28 \mathrm{MeV}$, which corresponds to the minimum of the curve in Fig. 1. For isotopes beyond $N=32$, appropriate extrapolations and larger model spaces are required and will be considered in future works.

The accuracy of the many-body truncation of the selfenergy at second order must also be assessed. To this extent, we consider the standard (Dyson) formulation of SCGF implemented within the third-order algebraic diagrammatic construction [ADC(3)], which goes beyond the full third order [26,27]. The comparison in closed-shell isotopes ${ }^{40} \mathrm{Ca}$, ${ }^{48} \mathrm{Ca}$, and ${ }^{52} \mathrm{Ca}$ (top panel of Fig. 2) shows that the correction from third- and higher-order diagrams is rather constant along the chain. Respectively, in $N_{\max }=9$ we obtain $E_{0}^{\mathrm{ADC}(3)-\text { Dys }}-$

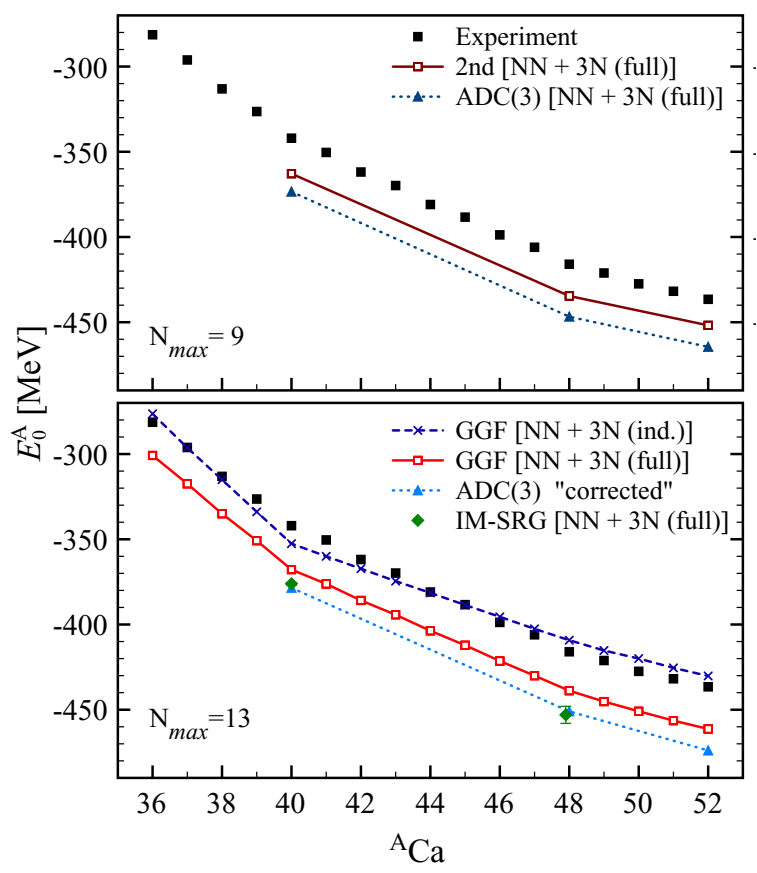

FIG. 2. (Color online) Experimental (solid squares) [28-30] and calculated ground-state energies of $\mathrm{Ca}$ isotopes. Top panel: Secondorder Gorkov and Dyson-ADC(3) results for ${ }^{40,48,52} \mathrm{Ca}$ obtained with a $N_{\max }=9$ model space and the full Hamiltonian. Bottom panel: Second-order Gorkov results with $N N$ plus induced (crosses) and $N N$ plus full (open squares) $3 N F s$ and $N_{\max }=13$. Full 3 NF Gorkov results corrected for the ADC(3) correlation energy extracted from the top panel (dotted line with solid triangles). IM-SRG results [12] are for the same $3 \mathrm{NF}$ and are extrapolated to infinite model space (diamonds with error bars).
$E_{0}^{2 \mathrm{nd}-\mathrm{Gkv}}=-10.6,-12.1$, and $-12.6 \mathrm{MeV}$, which correspond to $\approx 2.7 \%$ of the total binding energy. Assuming that these differences are converged with respect to the model space, we add them to our second-order Gorkov results with $N_{\max }=13$ and display the results in the bottom panel of Fig. 2. Resulting values agree well with IM-SRG calculations of ${ }^{40} \mathrm{Ca}$ and ${ }^{48} \mathrm{Ca}$ based on the same Hamiltonian [12]. This confirms the robustness of the present results across different many-body methods. The error due to missing induced 4NFs was also estimated in Ref. [12] by varying the SRG cutoff over a (limited) range. Up to $\approx 1 \%$ variations were found for masses $A \leqslant 56$ (e.g., less than $0.5 \%$ for ${ }^{40} \mathrm{Ca}$ and ${ }^{48} \mathrm{Ca}$ ) when changing $\lambda$ between 1.88 and $2.24 \mathrm{fm}^{-1}$. We take this estimate to be generally valid for all the present calculations.

A first important result of this work appears in the bottom panel of Fig. 2, which compares the results obtained with $N N$ plus induced $3 N F$ s and $N N$ plus full $3 N F s$. The trend of the binding energy of $\mathrm{Ca}$ isotopes is predicted incorrectly by the induced 3NFs alone. This is fully amended by the inclusion of leading chiral 3NFs. However, the latter introduce additional attraction that results in a systematic overbinding of ground-state energies throughout the whole chain. Analogous results are obtained for $\mathrm{Ar}, \mathrm{K}, \mathrm{Sc}$, and $\mathrm{Ti}$ isotopic chains (not shown here), leading to the same conclusion regarding the role of the initial chiral $3 \mathrm{NF}$ in providing the correct trend and in generating overbinding at the same time.

The $N N$ plus induced $3 \mathrm{~N}$ interaction, which originates from the $N N$-only $\mathrm{N}^{3} \mathrm{LO}$ potential, generates a wrong slope in Fig. 2 and exaggerates the kink at ${ }^{40} \mathrm{Ca}$. The corresponding two-nucleon separation energies are shown in Fig. 3 and are significantly too large (small) for $N \leqslant 20(N>20)$. Including chiral $3 \mathrm{NFs}$ corrects this behavior to a large extent and predicts $S_{2 n}$ close to the experiment for isotopes above ${ }^{42} \mathrm{Ca}$. Figure 3 also shows results for microscopic shell model [19,30] and coupled-cluster [9] calculations above ${ }^{41} \mathrm{Ca}$ and ${ }^{49} \mathrm{Ca}$, respectively, which are based on similar chiral forces. Our calculations confirm and extend these results within a full-fledged $a b$ initio approach for the first time.

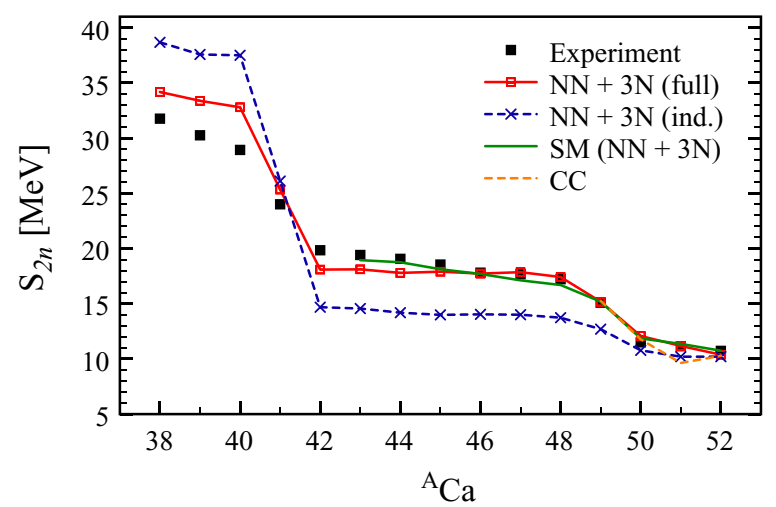

FIG. 3. (Color online) Two-nucleon separation energies, $S_{2 n}$, of $\mathrm{Ca}$ isotopes. Gorkov calculations are shown for the induced (crosses) and full (open squares) Hamiltonians and are compared to the experiment (solid squares) [28-30]. Results from shell-model calculations with chiral 3NFs (solid line) [19,30] and coupled cluster (dashed line) [9] are also shown. 
The $S_{2 n}$ jump between $N=20$ and $N=22$ is largely overestimated with the $N N$ plus induced $3 \mathrm{NFs}$, which confirms the findings of Refs. [8,31] based on the original $N N$ interaction. The experimental $Z=20$ magic gap across ${ }^{48} \mathrm{Ca}$ is $\Delta_{\pi}\left({ }^{48} \mathrm{Ca}\right) \equiv 2 E_{0}^{48} \mathrm{Ca}-E_{0}^{49} \mathrm{Sc}-E_{0}^{47} \mathrm{~K}=6.2 \mathrm{MeV}$, whereas it was found to be $10.5 \mathrm{MeV}$ in Ref. [31]. The magic gap is somewhat larger in the present calculations: it is equal to $16.5 \mathrm{MeV}$ with the $N N$ plus induced $3 \mathrm{NF}$ and is reduced to 12.4 MeV including the full 3NF.

The Koltun sum rule (3) computes the binding energy as a weighted sum of one-nucleon removal energies. The systematic overbinding observed in the present results thus relates to a spectrum in the A-1 system (not shown here) that is too spread out. This is reflected in the excessive distance between major nuclear shells or effective singleparticle energies (ESPE) [16,32]. In turn, the overestimated $N=20$ gap and the jump of the $S_{2 n}$ between $N=20$ and 22 relate to the exaggerated energy separation between $s d$ and $p f$ major shells generated by presently employed chiral interactions. Eventually, a too dilute ESPE spectrum translates into underestimated radii. Specifically, we obtain matter radii of $r_{\mathrm{rms}}^{\mathrm{GGF}}=2.89,2.94$, and $2.97 \mathrm{fm}$ for ${ }^{40} \mathrm{Ca},{ }^{44} \mathrm{Ca}$, and ${ }^{48} \mathrm{Ca}$, respectively, to be compared with experimental values of $r_{\text {rms }}^{\exp }=3.48,3.52$, and $3.48 \mathrm{fm} \mathrm{[33].}$

Presently, $\mathrm{ADC}(3)$-corrected energies with the $N N$ plus full $3 \mathrm{NFs}$ (Fig. 2) overbind ${ }^{40} \mathrm{Ca},{ }^{48} \mathrm{Ca}$, and ${ }^{52} \mathrm{Ca}$ by 0.90 , 0.73 , and $0.72 \mathrm{MeV} / \mathrm{A}$, respectively. It can be conjectured that such a behavior correlates with a predicted saturation point of symmetric nuclear matter that is too bound and located at too high density compared to the empirical point. Recent calculations of homogeneous nuclear matter based on chiral interactions $[2,3]$ predict a saturation point in the vicinity of the empirical point with an uncertainty that is compatible with the misplacement suggested by our analysis. However, such calculations use a different $3 \mathrm{NF}$ cutoff $\Lambda_{3 N}=$ $500 \mathrm{MeV}$ and different values of $c_{D}$ and $c_{E}$. Additional SCGF calculations like those in Ref. [3] but with the same $N N+3 N$ chiral interactions used here would help in confirming this conjecture.

The systematic of $S_{2 n}$ obtained with the $N N$ plus full $3 N F s$ is displayed in Fig. 4 along $\mathrm{Ar}, \mathrm{K}, \mathrm{Ca}, \mathrm{Sc}$, and Ti isotopic chains, up to $N=32$. When the neutron chemical potential lies within the $p f$ shell, predicted $S_{2 n}$ reproduce experiment to good accuracy. Still, the quality slightly deteriorates as the proton chemical potential moves down into the $s d$ shell, i.e., going from $\mathrm{Ca}$ to $\mathrm{K}$ and $\mathrm{Ar}$ elements. The increasing underestimation of the $S_{2 n}$ is consistent with a too large gap between proton $s d$ and $p f$ major shells that prevents quadrupole neutron-proton correlations to switch on. The too large jump of the $S_{2 n}$ between $N=20$ and $N=22$ is visible for all elements and becomes particularly pronounced as one moves away from the proton magic ${ }^{40} \mathrm{Ca}$ nucleus where the experimental jump is progressively washed out. At $N=18$, the situation deteriorates when going from ${ }^{38} \mathrm{Ca}$ to ${ }^{39} \mathrm{Sc}$ and ${ }^{40} \mathrm{Ti}$ (but not going to ${ }^{37} \mathrm{~K}$ and ${ }^{36} \mathrm{Ar}$ ), i.e. when the proton chemical potential moves up into the $p f$ shell. This is again consistent with an exaggerated shell gap between $s d$ and $p f$ shells that prevents neutron-proton correlations to switch on when the chemical potentials sit on both sides of the gap.

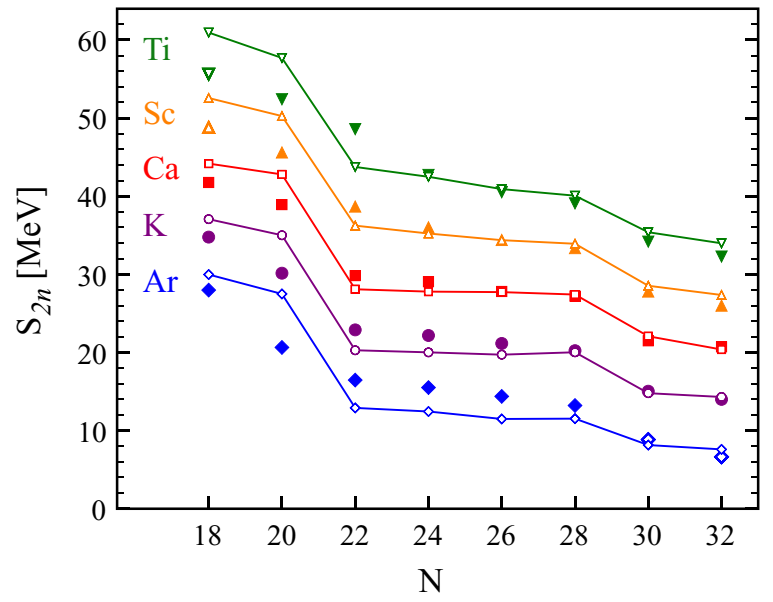

FIG. 4. (Color online) Two-neutron separation energies, $S_{2 n}$, along $\mathrm{Ar}, \mathrm{K}, \mathrm{Ca}, \mathrm{Sc}$, and $\mathrm{Ti}$ isotopic chains. The experimental values (solid symbols) [28-30] are compared to second-order Gorkov calculations with the $N N$ plus full $3 \mathrm{NFs}$ (solid lines). Values for $\mathrm{K}$, $\mathrm{Ca}, \mathrm{Sc}$, and Ti are, respectively, shifted by $+5,10,15$, and $20 \mathrm{MeV}$ for display purposes. Isolated open symbols are AME2012 extrapolations of experimental data [28].

One should note that ${ }^{46} \mathrm{Ti}$ and ${ }^{48} \mathrm{Ti}$ have the tendency to be slightly deformed in their intrinsic frame. While the associated enhanced quadrupole correlations are grasped through secondorder diagrams in the present symmetry-conserving approach, it is of interest to assess in the future the effects of an explicit coupling to collective quadrupole excitations or the breaking of rotational symmetry. Still, we do not expect this to alter any of our conclusions significantly.

Conclusions. We have reported on the first $a b$ initio description of complete isotopic chains in the medium-mass region of the nuclear chart. Exploiting the mechanism of symmetry breaking and keeping the simplicity of a singlereference scheme, the description of open-shell, i.e. near degenerate, nuclei is made possible thanks to the first-ever implementation of self-consistent Gorkov-Green's function technique on the basis of realistic two- and three-nucleon forces. This represents a qualitative breakthrough that opens up unprecedented possibilities regarding the number of nuclei reachable via ab initio methods. Such a development could be of interest to address other near-degenerate physical systems, e.g. open-shell molecules near equilibrium and/or molecules undergoing bond breaking, which are a current challenge for cutting-edge $a b$ initio methods in quantum chemistry (see e.g. Ref. [34]).

In this work we have focused on the ability of leading chiral 3NFs to describe absolute binding and two-neutron separation energies along $\mathrm{Ar}, \mathrm{K}, \mathrm{Ca}, \mathrm{Sc}$, and $\mathrm{Ti}$ chains, up to $N=32$. While available $N N+3 N$ chiral interactions typically perform well in the vicinity of oxygen isotopes, they were never tested for entire isotopic chains with $Z>9$. Leading $3 \mathrm{NFs}$ are found to be mandatory to reproduce the correct trend of binding energies for all isotopes, analogously to what was observed in lighter N, O, and F chains. Overall, the systematic of two-neutron separation energies is reproduced with a good quality. Still, absolute binding energies are systematically overestimated throughout the $Z \approx 20$ mass 
region and the magic character of $N=20$ and/or $Z=20$ nuclei is exaggerated. Preliminary results on the nickel chain [35] show a similar behavior for the $N=40$ closure, which reinforces our findings and points towards evident deficiencies of the employed $N N+3 N$ chiral forces. Within the present theoretical scheme, these defects can be traced back to the fact that deeply bound effective single-nucleon shells are too spread out and that the distance between major shells is too pronounced. It is conjectured that this relates to a saturation point of symmetric nuclear matter located at a slightly too large binding energy/density compared to the empirical point. We conclude that $a b$ initio many-body calculations of mediummass isotopic chains provide a stringent testing ground for modern theories of elementary nuclear interactions.
This work was supported by the DFG through Grant No. SFB 634; by the Helmholtz Alliance Program, Contract No. HA216/EMMI; by the United Kingdom Science and Technology Facilities Council (STFC) under Grants No. ST/J000051/1 and No. ST/L005816/1; and by the Natural Sciences and Engineering Research Council of Canada (NSERC), Grant No. 401945-2011. TRIUMF receives funding via a contribution through the National Research Council Canada. Calculations were performed using HPC resources from GENCI-CCRT (Grant No. 2013-050707) and the DiRAC Data Analytic System at the University of Cambridge (BIS National Einfrastructure Capital Grant No. ST/K001590/1 and STFC Grants No. ST/H008861/1, No. ST/H00887X/1, and No. $\mathrm{ST} / \mathrm{K} 00333 \mathrm{X} / 1)$.
[1] V. Somà and P. Bożek, Phys. Rev. C 78, 054003 (2008).

[2] K. Hebeler, S. K. Bogner, R. J. Furnstahl, A. Nogga, and A. Schwenk, Phys. Rev. C 83, 031301 (2011).

[3] A. Carbone, A. Polls, and A. Rios, Phys. Rev. C 88, 044302 (2013).

[4] E. Epelbaum, H.-W. Hammer, and U.-G. Meissner, Rev. Mod. Phys. 81, 1773 (2009).

[5] T. Otsuka, T. Suzuki, J. D. Holt, A. Schwenk, and Y. Akaishi, Phys. Rev. Lett. 105, 032501 (2010).

[6] H. Hergert, S. Binder, A. Calci, J. Langhammer, and R. Roth, Phys. Rev. Lett. 110, 242501 (2013).

[7] A. Cipollone, C. Barbieri, and P. Navrátil, Phys. Rev. Lett. 111, 062501 (2013).

[8] C. Barbieri and M. Hjorth-Jensen, Phys. Rev. C 79, 064313 (2009).

[9] G. Hagen, M. Hjorth-Jensen, G. R. Jansen, R. Machleidt, and T. Papenbrock, Phys. Rev. Lett. 109, 032502 (2012).

[10] R. Roth, S. Binder, K. Vobig, A. Calci, J. Langhammer, and P. Navrátil, Phys. Rev. Lett. 109, 052501 (2012).

[11] K. Tsukiyama, S. K. Bogner, and A. Schwenk, Phys. Rev. Lett. 106, 222502 (2011).

[12] H. Hergert, S. K. Bogner, S. Binder, A. Calci, J. Langhammer, R. Roth, and A. Schwenk, Phys. Rev. C 87, 034307 (2013).

[13] T. A. Lähde, E. Epelbaum, H. Krebs, D. Lee, U.-G. Meißner, and G. Rupak, Phys. Lett. B 732, 110 (2014).

[14] S. Binder, J. Langhammer, A. Calci, P. Navrátil, and R. Roth, Phys. Rev. C 87, 021303 (2013).

[15] S. Binder, J. Langhammer, A. Calci, and R. Roth, arXiv:1312.5685.

[16] V. Somà, T. Duguet, and C. Barbieri, Phys. Rev. C 84, 064317 (2011).

[17] V. Somà, C. Barbieri, and T. Duguet, Phys. Rev. C 87, 011303 (2013).
[18] V. Somà, C. Barbieri, and T. Duguet, Phys. Rev. C 89, 024323 (2014).

[19] J. D. Holt, T. Otsuka, A. Schwenk, and T. Suzuki, J. Phys. G 39, 085111 (2012).

[20] A. Carbone, A. Cipollone, C. Barbieri, A. Rios, and A. Polls, Phys. Rev. C 88, 054326 (2013).

[21] T. Duguet, P. Bonche, P. H. Heenen, and J. Meyer, Phys. Rev. C 65, 014311 (2001).

[22] E. D. Jurgenson, P. Navrátil, and R. J. Furnstahl, Phys. Rev. Lett. 103, 082501 (2009).

[23] D. R. Entem and R. Machleidt, Phys. Rev. C 68, 041001 (2003).

[24] R. Machleidt and D. R. Entem, Phys. Rep. 503, 1 (2011).

[25] P. Navrátil, Few-Body Systems 41, 117 (2007).

[26] J. Schirmer, L. S. Cederbaum, and O. Walter, Phys. Rev. A 28 , 1237 (1983).

[27] C. Barbieri, D. Van Neck, and W. H. Dickhoff, Phys. Rev. A 76, 052503 (2007).

[28] M. Wang, G. Audi, A. Wapstra, F. Kondev, M. MacCormick, X. Xu, and B. Pfeiffer, Chin. Phys. C 36, 1603 (2012).

[29] A. T. Gallant, J. C. Bale, T. Brunner, U. Chowdhury, S. Ettenauer, A. Lennarz, D. Robertson, V. V. Simon, A. Chaudhuri, J. D. Holt, A. A. Kwiatkowski, E. Mané, J. Menéndez, B. E. Schultz, M. C. Simon, C. Andreoiu, P. Delheij, M. R. Pearson, H. Savajols, A. Schwenk, and J. Dilling, Phys. Rev. Lett. 109, 032506 (2012).

[30] F. Wienholtz, D. Beck, K. Blaum, C. Borgmann, M. Breitenfeldt et al., Nature (London) 498, 346 (2013).

[31] C. Barbieri, Phys. Rev. Lett. 103, 202502 (2009).

[32] T. Duguet and G. Hagen, Phys. Rev. C 85, 034330 (2012).

[33] H. D. Wohlfahrt, E. B. Shera, M. V. Hoehn, Y. Yamazaki, and R. M. Steffen, Phys. Rev. C 23, 533 (1981).

[34] A. G. Taube, Mol. Phys. 108, 2951 (2010).

[35] V. Somà, C. Barbieri, A. Cipollone, T. Duguet, and P. Navrátil (unpublished). 